\section{Brain, Behavior and Evolution}

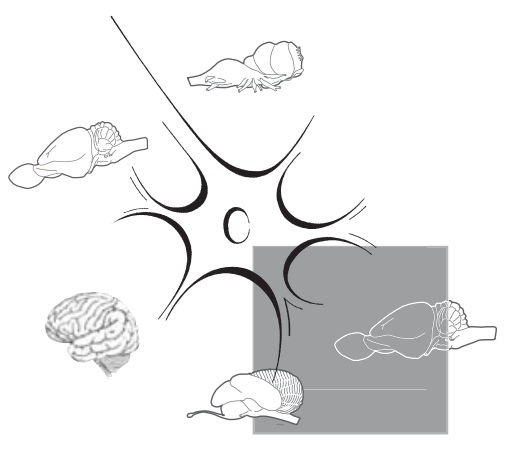

Brain Behav Evol 2015;85:1-3

DOI: $10.1159 / 000375438$
Published online: March 7, 2015

\title{
Even the Smallest Mammalian Brain Has Yet to Reveal Its Secrets
}

\author{
Robert K. Naumann \\ Animal Physiology/Systems Neurobiology and Neural Computation, \\ Humboldt University Berlin, Berlin, Germany
}

The evolution and function of the cortex remain an enduring mystery in the neurosciences. Some animals, such as bats and star-nosed moles, exhibit remarkable specializations in their sensory organs, which are reflected in specialized cortical architecture. Thus, studying unusual species may reveal aspects of cortical circuit function and evolution.

The Etruscan shrew is the smallest terrestrial mammal and, with a weight of only $64 \mathrm{mg}$, has one of the smallest brains of all mammals. Shrews show a wide range of social and exploratory behaviors as well as sophisticated prey-capture capabilities and unique adaptations of the cardiovascular and respiratory systems to small body size [Brecht et al., 2011]. I wanted to know whether such a small animal would still have the complex cortical architecture and multiple cortical areas found in larger mammals. As shown in a sagittal section through the shrew brain that had been stained for myelinated fibers (fig. 1a), histochemical staining methods revealed a number of well-defined regions and areas in the Etruscan shrew's cerebral cortex. For example, the somatosensory cortex is a large, myelin-rich region in the center whereas the visual cortex is a much smaller area posterior to the somatosensory cortex. These proportions correlate with the size of the cortical fields as assessed by microelectrode mapping [Roth-Alpermann et al., 2010] and reflect the differences in size of the cranial nerves [Brecht et al., 2011]. Multiple staining methods revealed a map of about 15 cortical regions and areas with similar cytoarchitectonic and histochemical features as in larger mammals [Naumann et al., 2012]; a number of them are shown as a flat-map of the cortex in figure $1 \mathrm{~b}$. Using this map, I counted up neuron numbers in all of the cortical regions and found that the visual cortex contains only about 40,000 neurons whereas the somatosensory cortex contains about 200,000; this is consistent with the Etruscan shrew's heavy reliance on whisker touch for prey recognition. In total, there are about $1,000,000$ neurons in each cortical hemisphere.

The Etruscan shrew's cortex is probably the smallest of all mammals - it is certainly unusually small. Thus, the brain of the last common ancestor of all mammals was likely significantly larger than that of the Etruscan shrew and, unlike most mammals, the Etruscan shrew's cerebral cortex has decreased significantly in size from that of its ancestors. Even with such a small cortex, the Etruscan shrew retains several aspects of the unique complexity of mammalian cortical circuits, such as a well-differentiated laminar structure and a relatively large number of cortical areas, yet the cortical thickness is remarkably reduced. In fact, the cortex is, on average, less than $500 \mu \mathrm{m}$ thick, which makes it possible to record activity from entire cortical columns in vivo using 2 -photon imaging. It may even be possible to record the activity of all neurons in one of the smaller areas such as the visual cortex. Thus, using a novel animal model may offer advantages for recording activity exhaustively in mammalian cortical circuits and discovering how structure-function relationships evolved. However, the mapping of areas and regions is only the first step towards understanding the mysterious machine that is the cortex. To reveal its secrets, techniques to study single-neuron and circuit function are necessary, as illustrated for the entorhinal cortex below.

Columns and modules are present in many cortical areas, but their function has remained elusive. In layer 2 of the medial entorhinal cortex of the rat, calbindin-positive cells form modules. Figure 1c shows a map of the surface of the medial entorhinal cortex and adjacent parasubiculum; green dots indicate the distribution of clusters of calbindin-positive cells (patches) and blue lines indicate the main direction of myelinated fibers at the border of layers 1 and

\section{KARGER 125}

C 2015 S. Karger AG, Basel

0006-8977/15/0851-0001\$39.50/0
Robert K. Naumann

Max Planck Institute for Brain Research

Max-von-Laue-Strasse 4

DE-60438 Frankfurt am Main (Germany)

www.karger.com/bbe 


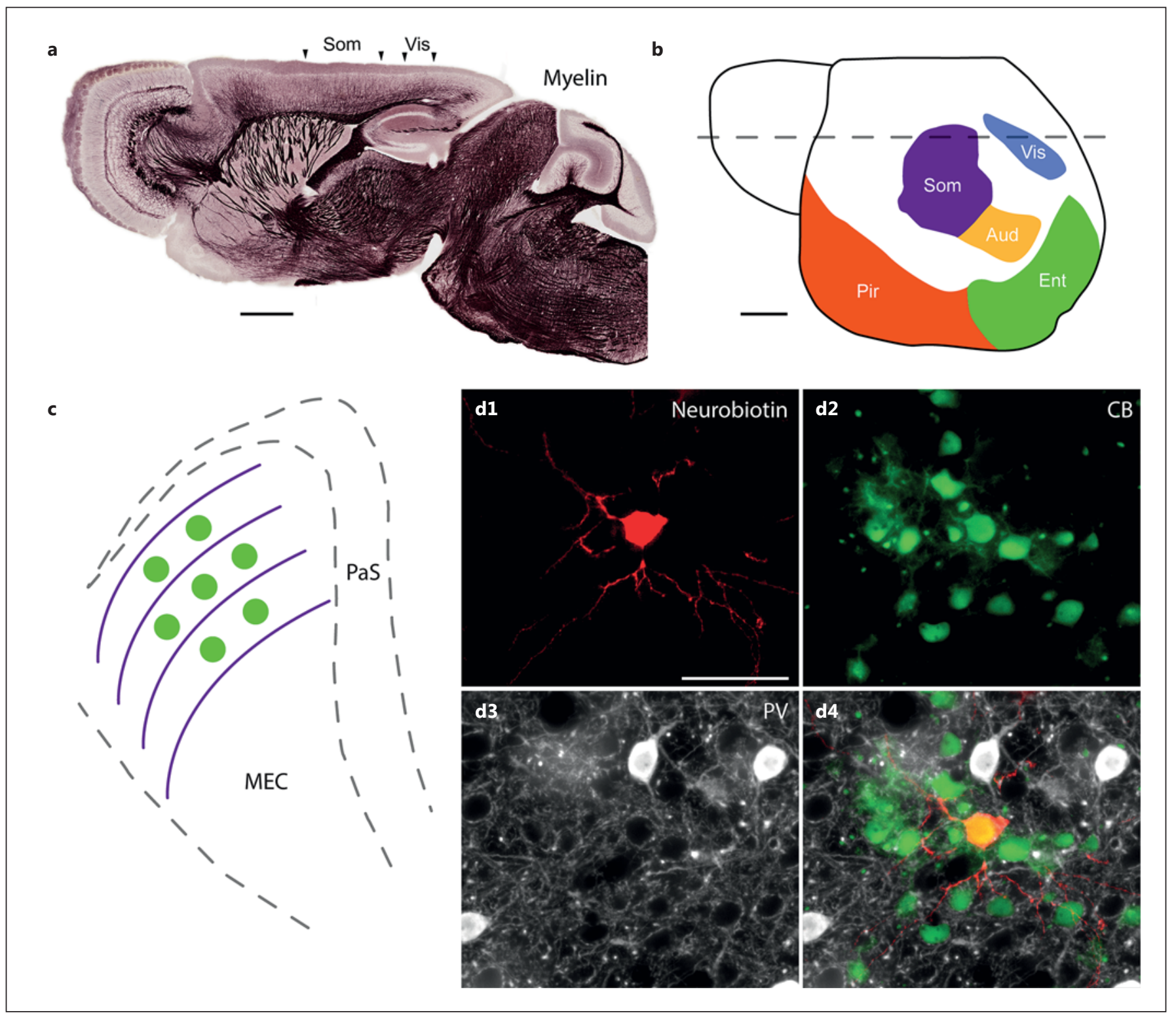

Fig. 1. Cortical regions in the Etruscan shrew and modules in the rat medial entorhinal cortex. a Sagittal section through the brain of the Etruscan shrew stained for myelinated fibers. $\mathbf{b}$ Mapping of some cortical areas on the surface of the Etruscan shrew cortex. Dashed line indicates sagittal section in a. c Surface map of the rat entorhinal cortex; area borders are indicated with dashed lines. Green dots show calbindin patches and blue lines the dominant orientation of myelinated fibers. $\mathbf{d}$ Immunohistochemical staining of an identified cell (d1) allows locating it within a patch of calbindin-positive cells (d2) and shows that the cell was immunonegative for parvalbumin (d3) but immunopositive for calbindin as shown in the overlay (d4). Aud = Auditory cortex; $\mathrm{CB}=$ calbindin; Ent = entorhinal cortex; $\mathrm{MEC}=$ medial entorhinal cortex; $\mathrm{PaS}=$ parasubiculum; $\mathrm{Pir}=$ piriform cortex; $\mathrm{PV}=$ parvalbumin; Som = somatosensory cortex; Vis = visual cortex. Scale bars: 500 $\mu \mathrm{m}(\mathbf{a}), 1 \mathrm{~mm}(\mathbf{b}), 50 \mu \mathrm{m}(\mathbf{d})$.
2. Layer 2 also has the highest density of 'grid cells', i.e. cells that fire in a hexagonal pattern tiling space when an animal explores the environment. Grid cells have been described in the entorhinal cortex of rodents, bats, monkeys and humans, and they likely function in orientation and navigation. Interestingly, grid cells form functional modules in layer 2 of the medial entorhinal cortex; this led our group to propose that calbindin-positive cells may be involved in generating the firing patterns of grid cells [Brecht et al., 2013]. There are two principal cell types in layer 2 of the rat medial entorhinal cortex: stellate cells and calbindin-positive pyramidal cells. Further anatomical studies showed that the calbindin patches strongly overlap with cholin- 
ergic axons, which are implicated in the generation of the theta rhythm. Next, we recorded activity from identified cells in freely moving or anaesthetized animals, followed by immunohistochemical detection of cell types. This allowed us to correlate physiological properties with neuron morphology and cell-type markers (fig. 1d). We discovered that calbindinpositive cells - like grid cells - were more strongly theta-modulated than calbindinnegative cells [Ray et al., 2014]. Further recordings of anatomically identified cells in freely moving animals suggest that the calbindin-positive cells themselves are more often grid cells than the other cells in layer 2 of the medial entorhinal cortex [Tang et al., 2014].

Several types of commonly investigated cortical modules, such as barrels in ro- dents or orientation columns, are present in only a limited number of mammals. Since grid cells have been found in species like bats and monkeys, separated for a long time in evolutionary history, we are currently examining whether entorhinal modular structures are broadly conserved across mammals or are more limited in their phylogenetic distribution.

\section{References}

Brecht M, Naumann R, Anjum F, Wolfe J, Munz M, Mende C, Roth-Alpermann C (2011): The neurobiology of Etruscan shrew active touch. Philos Trans R Soc Lond B Biol Sci 366:3026-3036.

Brecht M, Ray S, Burgalossi A, Tang Q, Schmidt H, Naumann R (2013): An isomorphic mapping hypothesis of the grid representation. Philos Trans R Soc Lond B Biol Sci 369: 20120521.

-Naumann RK, Anjum F, Roth-Alpermann C, Brecht M (2012): Cytoarchitecture, areas, and neuron numbers of the Etruscan shrew cortex. J Comp Neurol 520:2512-2530.
Ray S, Naumann R, Burgalossi A, Tang Q, Schmidt H, Brecht M (2014): Grid-like arrangement and theta-modulation of a pyramidal cell microcircuit in layer 2 of medial entorhinal cortex. Science 343:891-896.

Roth-Alpermann C, Anjum F, Naumann R, Brecht M (2010): Cortical organization in the Etruscan shrew (Suncus etruscus). J Neurophysiol 104:2389-2406.

- Tang Q, Burgalossi A, Ebbesen C, Ray S, Naumann R, Spicher D, Schmidt H, Brecht M (2014): Pyramidal and stellate cell-specificity of grid and border representations in layer 2 medial entorhinal cortex. Neuron 84:11911197. 